\title{
Crushing Pain
}

National Cancer Institute

\section{Source}

National Cancer Institute. Crushing Pain. NCI Thesaurus. Code C101174.

A sense of discomfort or distress that is squeezing or excessively compressing. 